\title{
CALCULATION AND ANALYSIS ON SCATTERING CHARACTERISTICS OF NON- SPHERICAL PARTICLES OF HAZE
}

\author{
Wenming Ji ${ }^{1, *}$, Fuyuan Jiang ${ }^{2,3,4}$, Chenxi Chu ${ }^{2,3,4}$ \\ ${ }^{1}$ School of Meteorology and Oceanography,National Defense Science and Technology University,Changsha,China - \\ jiwenming17@mail.nudt.edu.cn \\ ${ }^{2}$ Collaborative Innovation Center on Forecast and Evaluation of Meteorological Disasters, Nanjing University of Information \\ Science and Technology(NUIST), Nanjing, China-sev7enjj@126.com \\ ${ }^{3,4}$ Key Laboratory for Aerosol-Cloud-Precipitation of China Meteorological Administration, Key Laboratory of Meteorological \\ Disaster of Ministry of Education, NUIST, Nanjing, China-1179917545@qq.com
}

KEY WORDS: T-Matrix; Non-spherical; Scattering; Depolarization; Haze

\begin{abstract}
:
The light scattering characteristics of sulfate, one of the main pollutant particles in haze, are calculated by T-Matrix method at a target wavelength of $550 \mathrm{~nm}$. The variation between shape factors (such as effective radius and aspect ratio) and scattering phase functions with different types and shapes are analysed in small scale range. The influence of shape factors on scattering cross section and depolarization ratio of particles are also discussed. Results show that the shape of particles has great effects on the spatial distribution of scattering energy, and the scattering properties of particles are sensitive to aspect ratio. The depolarization of spherical particles is close to zero, while the difference between ellipsoidal and cylindrical particles reaches several orders of magnitude. When the equivalent radius is larger than $1.0 \mu \mathrm{m}$, the mean depolarization ratio of the non-spherical particles is greater than 0.2. The mean depolarization ratio and scattering cross section of non-spherical particle change continuously with a certain aspect ratio and particle size range, and the shape of some particles can be therefore distinguished under certain conditions.
\end{abstract}

\section{INTRODUCTION}

The haze phenomenon is caused by the accumulation of primary pollutants in the atmosphere and secondary pollutants formed by chemical reactions under certain atmospheric capacity (He at al. 2013; Wu, 2012). Chow et al. (2002) found that the contribution of fine particles $(\mathrm{D}<2.5 \mu \mathrm{m})$ to scattering is

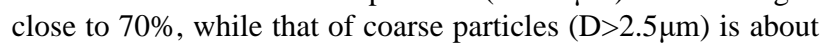
$30 \%$. The concentration of PM2.5 tends to stay at a high level during haze event. The important chemical components of PM2.5 are secondary water-soluble particles and organic matter, mainly including ammonium sulfate, sulfuric acid, ammonium nitrate and carbon aerosols. Therefore, the typical factor leading to haze event is the concentration of fine particles, especially the concentration of sulfate and nitrate, as well as the relative humidity of the atmosphere. Yang et al. (2011) found that sulfate and nitrate accounted for 7.1 57.0\% of the chemical constituents of PM2.5 in some cities of China.

At present, researches mainly focus on the subjects of the physical and chemical components, optical properties and causes of haze by means of ground-based observation, meteorological analysis, aerosol particle transformation, remote sensing inversion and model simulation. Zhang et al. (2019) analyzed a typical haze event in June 2018 Nanjing, China by using both ground-based and spaceborne sensors, combined with sounding and HYSPLIT backward trajectory data, where data from different sensors exhibited a good consistency. A polarized optical particle counter (POPC) has been developed by both the Beijing Institute of Atmospheric Physics and Chinese Academy of Sciences to measure the morphological characteristics of aerosols. It can distinguish aerosol types by polarization information of scattered light (Tian et al. 2018). Accordingly, the theoretical analysis of the scattering characteristics of non-spherical particles, especially the characteristics of depolarization, is helpful to improve the application ability of this kind of instrument. Furthermore, The shape of aerosol particles is also complex and diverse. Generally, particles are treated as spheres, which will inevitably lead to errors. Many works are trying to solve the radiation characteristics of non-spherical aerosol particles with various numerical simulation methods.

In this paper, the scattering characteristics of non-spherical particles of haze are simulated by using T-matrix method. The scattering characteristics of non-spherical particles, including ellipsoidal, cylindrical and Chebyshev particles, are simulated and calculated for sulfate, one of the main pollutant in haze at a target wavelength of $550 \mathrm{~nm}$. The changes of forward and backward scattering, scattering cross section and depolarization with aspect ratio and equivalent radius, and that of scattering phase function with scattering angle are analyzed.

\section{T-MATRIX METHOD}

T-matrix method is a powerful tool for calculating the scattering characteristics of aspheric particles. It only depends on the physical and geometrical characteristics of particles, including refractive index, size and shape, but has nothing to do with the incident field (Bohren and Huffman, 1983). This method has high accuracy in calculating the scattering characteristics of small particles and is often used as a standard for testing other methods. In this method, in order to describe the light scattering from an arbitrary non-spherical particle, a spherical coordinate

\footnotetext{
* Corresponding author - Wenming Ji
} 
system of particles with origin inside the particle is adopted. Both the incident field outside the tangential sphere of the scattered and the scattered field inside the tangential sphere of the scattered can be expressed respectively by the regular vector wave function and the vector wave function(Mishchenko et al. 2002).

$$
\begin{aligned}
E^{i n c}(r) & =\sum_{n=1}^{n_{\max }} \sum_{m=-n}^{n}\left\{a_{m n} \operatorname{Rg}\left(M_{m n}(k r)\right)+b_{m n} \operatorname{Rg}\left(N_{m n}(k r)\right)\right\} \\
E^{s c a}(r) & =\sum_{n=1}^{n_{\max }} \sum_{m=-n}^{n}\left\{p_{m n} M_{m n}(k r)+q_{m n} N_{m n}(k r)\right\},|r|>r_{0}
\end{aligned}
$$

Where $\operatorname{Rg}\left(M_{m n}(k r)\right)$ and $\operatorname{Rg}\left(N_{m n}(k r)\right)$ represent positive values of $M_{m n}(k r)$ and $N_{m n}(k r)$ respectively. The scattering coefficients $p_{m n}$ and $q_{m n}$ and incident wave coefficients $a_{m n}$ and $b_{m n}$ are linearly correlated and can be described in the form of matrix (Mishchenko et al. 1996):

$$
\left[\begin{array}{l}
p \\
q
\end{array}\right]=T\left[\begin{array}{l}
a \\
b
\end{array}\right]=\left[\begin{array}{ll}
T^{11} & T^{12} \\
T^{21} & T^{22}
\end{array}\right]\left[\begin{array}{l}
a \\
b
\end{array}\right]
$$

The above formula is the basic equation of T-matrix method, $a_{m n}$ and $b_{m n}$ can be calculated by approximate analytic form.

In order to fully describe electromagnetic scattering, both intensity and polarization must be considered. For a beam with intensity and polarization, the intensity and polarization state can be described by Stokes vector $I=\{I, Q, U, V\}$. The parameter $I$ is proportional to the beam flux, and the parameters $Q$ and $U$ describe the linear polarization of the beam, while the parameter $V$ describes the circular polarization. The Stokes vectors of incident and scattered beams are connected by a scattering matrix $F$ of $4 * 4$. Matrix $\mathrm{F}$ can be calculated by $\mathrm{T}$ matrix method. $R$ represents the distance from the particle to the detector, $N_{0}$ represents the number density of particles, $V$ represents the wave number. For each scattering angle $\theta$, there is the following formula (Massic et al. 2012):

$$
I^{\mathrm{sca}}=\frac{C_{s c a} n_{0} d v}{4 \pi R^{2}} F(\theta) I^{i n c}
$$

The scattering matrix of rotationally symmetric particles can be simplified to:

$$
F(\theta)=\left[\begin{array}{cccc}
a_{1}(\theta) & b_{1}(\theta) & 0 & 0 \\
b_{1}(\theta) & a_{2}(\theta) & 0 & 0 \\
0 & 0 & a_{3}(\theta) & b_{2}(\theta) \\
0 & 0 & -b_{2}(\theta) & a_{4}(\theta)
\end{array}\right]
$$

In the formula above, $F(\theta)$ is the scattering phase function, $\theta$ is the scattering angle and satisfies the following relationship:

$$
\frac{1}{2} \int_{0}^{\pi} d \theta \sin \theta a_{1}(\theta)=1
$$

Generally, the phase functions in different scattering angles can be integrated by the following formula to represent the energy change of particles:

$$
F_{\text {int }}=\int_{\theta_{1}}^{\theta_{2}} a_{1}(\theta) \sin \theta \cos \theta d \theta
$$

When the polarization of incident light is parallel to the scattering plane, the depolarization ratio of particles can be written as follows:

$$
\delta_{H}=\frac{a_{1}(\theta)-a_{2}(\theta)}{a_{1}(\theta)+2 b_{1}(\theta)+a_{2}(\theta)}
$$

After the depolarization values of phase functions and each scattering angle are obtained, the mean depolarization ratio of particles with scattering angle $\theta_{1}$ and $\theta_{2}$ can be calculated:

$$
\bar{\delta}_{H}=\frac{1}{2} \int_{\theta_{1}}^{\theta_{2}} d \theta \sin \theta a_{1}(\theta) \delta_{H}(\theta)
$$

The average scattering cross sections from $\theta_{1}$ to $\theta_{2}$ can be expressed as:

$$
\bar{C}_{s c a}=\frac{1}{2} C_{s c a} \int_{\theta_{1}}^{\theta_{2}} d \theta \sin \theta a_{1}(\theta)
$$

\section{ANALYSIS OF SCATTERING CHARACTERISTICS OF NON-SPHERICAL PARTICLES}

Because the complex refractive index of aerosol particles is difficult to be accurately determined, this paper mainly focuses on the reference data provided by HITRAN 2012 database (Mishchenko and Travis,1998). Chen et al. found that SO42- is a key factor in formation of haze (Xu, 2003). Thus, when discussing the scattering characteristics of single particle, sulfate particle is a good example for our calculation because it has three representative shapes including ellipsoid, cylinder and Chebyshev (Massic and Herving, 2013).The shape factors mainly considered in the simulation are both the aspect ratio and equivalent radius describing non-sphericity of particle. The radius of non-spherical particle is approximated by the radius of sphere with equal surface area. As shown in Figure 1, for a rotating ellipsoid particle, the ellipsoid rotates along the Z-axis, $a$ and $\mathrm{b}$ denote the corresponding short axis and long axis respectively. Its aspect ratio can be expressed as the ratio of both axis lengths, i.e. a/b. For a finite cylinder with the same rotating axis, its aspect ratio can be expressed as $D / L$ where $L$ denotes the corresponding generatrix length and $D$ represents the diameter of the cylinder. For Chebyshev particle with invariant rotation axis, the shape factor can be expressed by effective radius, deformation parameter $\varepsilon$ and polynomial $n$. If the aspect ratios of two particles are equal, they are regarded as the same non-sphericity.

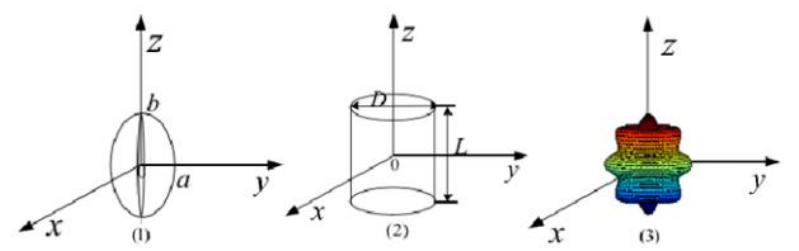

Figure 1. Three typical non-spherical models

\subsection{Scattering phase function of particles with different forms}

Figure 2 shows distributions of scattering phase function with regard to scattering angle for six different shapes. It is noted that logarithmic transformation is used for scattering energy. 
In general, most of the scattering energy is concentrated in the main lobe area of forward scattering. While the side scattering is the weakest, and the backscattering is slightly stronger than the side scattering. This distribution is similar to that calculated by Mie scattering. This shows that the energy
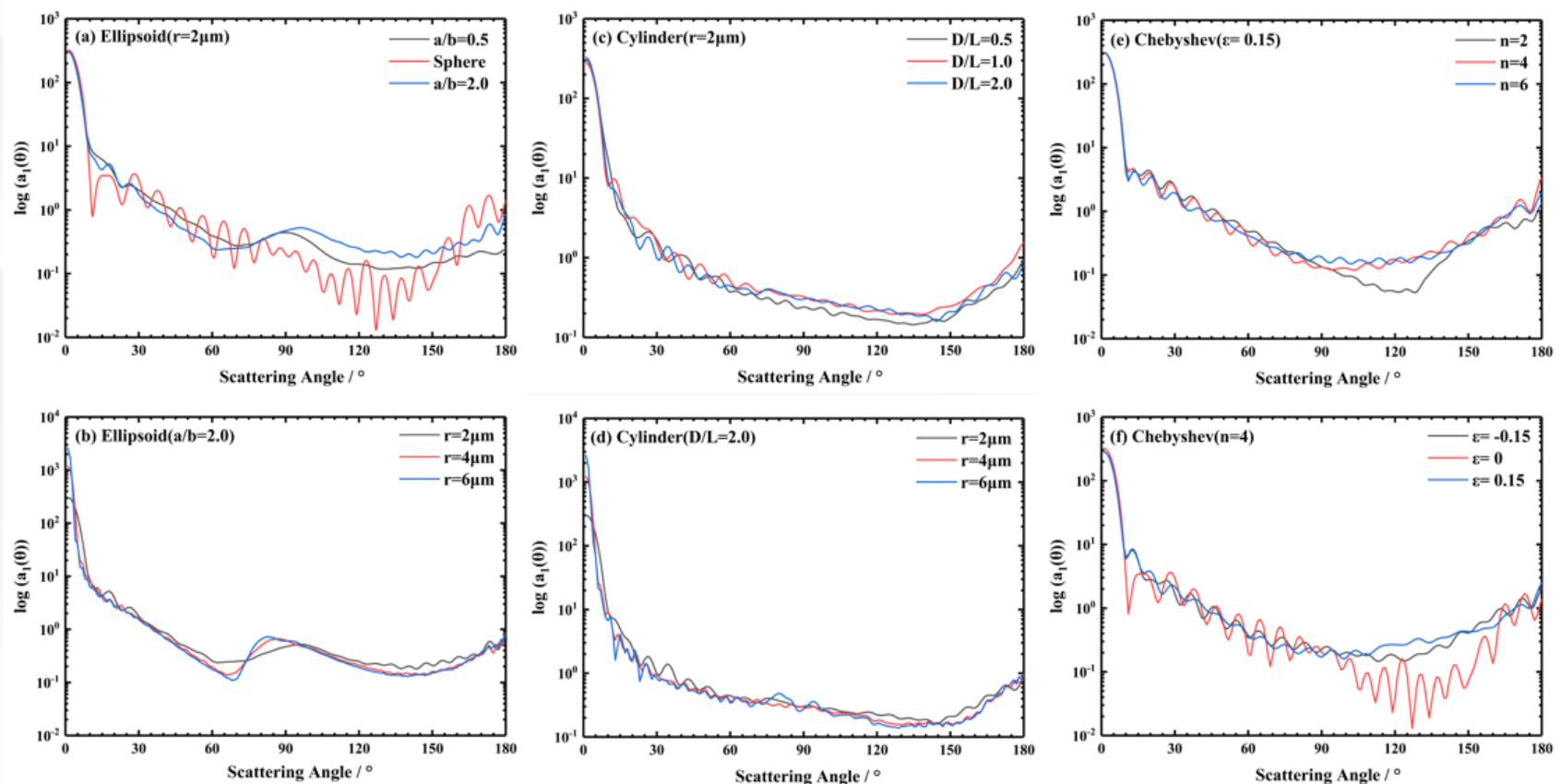

Figure 2. The distributions of scattering phase function with regard to scattering angle for six different shapes. It is noted that logarithmic transformation is used for scattering energy. (a) Phase function of ellipsoidal particles with different aspect ratio(r=2 $\mu \mathrm{m})$.

(b)Phase function of ellipsoidal particles with different equivalent radius $(\mathrm{a} / \mathrm{b}=2)$. (c) Phase function of cylindrical particles with

different aspect ratio( $\mathrm{r}=2 \mu \mathrm{m})$. (d)Phase function of cylindrical particles with different equivalent radius(D/L=2). (e) Phase function of different polynomials of Chebyshev particles $(\mathrm{r}=2 \mu \mathrm{m}, \varepsilon=0.15)$. (f) Phase function of different deformation parameters of Chebyshev particles $(\mathrm{r}=2 \mu \mathrm{m}, \mathrm{n}=4)$.

distribution of forward scattering is stronger than that of backscattering that is more significant to be detection. For ellipsoidal particles with different aspect ratios $(a / b)$, the distribution of scattering energy varies greatly with regard to different scattering angles $\left(100^{\circ} \sim 180^{\circ}\right)$. The phase function of spherical particles is more volatile than that of particles with other shapes, while the trend of particles with the same nonsphericity is similar (Figure 2a). For cylindrical particles with different aspect ratios $(D / L)$, they are slightly different from ellipsoidal particles. The fluctuation amplitude of the phase function for cylindrical particles is weak and the whole trend is relatively stable. It indicates that the scattering characteristics of cylindrical particles are not sensitive to the shape factor (Figure 2c). For ellipsoids (Figure 2b) and cylindrical particles (Figure $2 d)$ with different equivalent radius, when the equivalent radius changes from 2 to $6 \mu \mathrm{m}$, the phase function of forward scattering changes by nearly one order of magnitude. The decline rate of phase function in the main lobe region will increase with the increasing forward scattering ability of particles. The trend of phase function for ellipsoidal particles is also obviously different from cylindrical particles. The phase function for ellipsoidal particles has a maximum at $90^{\circ}$, while that for cylindrical particles does not show a significant maximum but a stable trend. In general, it is noted that the relationship between the scattering intensity of the forward main lobe and the distortion degree of the particle shape is not a regular monotonous change.

Figures 2ef show the distributions of phase function with regard to scattering angle for Chebyshev particles with different order number and deformation parameters, respectively. With the increase of order $n$, the energy distribution of forward scattering is more concentrated than that of the first two kinds of particles. However, from the change range of phase function, forward scattering is not sensitive to the change of order. When the deformation parameter $\varepsilon$ is fixed for the sphere, the larger the order $n$ is, the closer the particles are and the smaller the shape change is. The order has a significant influence on the lateral scattering and backscattering for Chebyshev particles, and the change of backscattering is close to one order of magnitude. Similar results can be obtained when the deformation parameter $\varepsilon$ is fixed, but the difference is that the fluctuation of backscattering energy is larger than that of the lateral scattering.

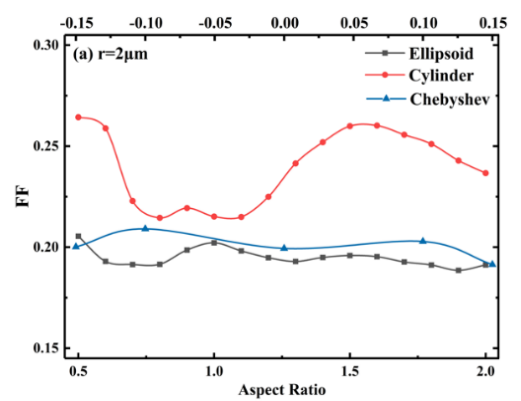




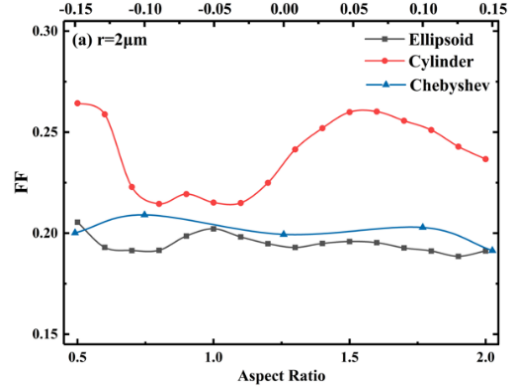

Figure 3. The integral forward scattering and back-scattering phase function relationship with aspect $\operatorname{ratio}(r=2 \mu \mathrm{m})$ of sulfate particle. (a) forward scattering phase function; (b)backscattering phase function

The forward scattering and backscattering of particles have special significances for laser transmission and lidar detection. Based on the requirements for the forward and backward detection range of the scattering cloud particles detector proposed by Baumgardner in 2001 and POPC, the forward scattering $\left(4^{\circ} \sim 14^{\circ}\right)$ and backscattering $\left(146^{\circ} \sim 176^{\circ}\right)$ phase functions of different sulfate particle forms are integrated according to the Eq. (9), where the forward and backscattering phase functions are expressed by $F F$ and $B F$, respectively. The size and shape of particle can be judged by forward scattering and backward polarization.

Figure $3 \mathrm{a}$ shows the relationship between the integral of the forward scattering phase function and the aspect ratio of particles with different morphologies. When the range of aspect ratio varies from 0.5 to 1.0 , the forward scattering phase functions for both ellipsoidal and cylindrical particles decrease firstly and then increase with the increaseing aspect ratio. In the range of 1 to 2.0 for aspect ratio, the forward scattering phase functions of ellipsoidal particles fluctuate slightly, while that of cylindrical particles increase firstly and then decrease. In the specific range of aspect ratio, the forward scattering phase function of ellipsoidal particles fluctuates around 0.19, while that of cylindrical particles fluctuates between 0.21 and 0.26 , with a mean value of 0.24 . The phase function of Chebyshev particles is slightly larger than that of ellipsoidal particles, and the overall fluctuation is relatively stable. Therefore, in a certain range of aspect ratio, the forward scattering phase function of cylindrical particles with different shapes is the largest, followed by that of Chebyshev particles and ellipsoidal particles. Figure 3b shows the relationship between backward scattering phase function and aspect ratio of particles for different shapes. In the direction of backscattering, the relationship between the scattering phase function and the aspect ratio of particles with different morphologies is more complex than that in the direction of forward scattering, which is difficult to be used as reference data.

\subsection{Depolarization characteristics of particles with different morphologies}

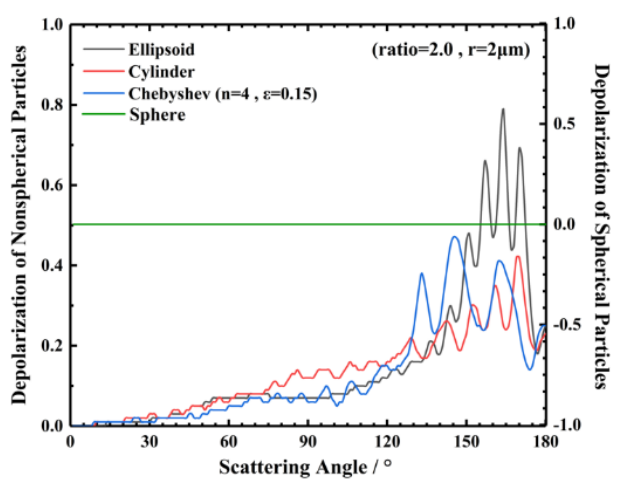

Figure 4. Depolarization of three different shapes of particles at different scattering angles.

The depolarization of particles can also can also reflect some information on particle shape to some extents. Figure 4 shows the distribution of depolarization ratios with scattering angles for particles of different shapes with an equivalent radius of 2 $\mu \mathrm{m}$. It can be found that the non-spherical particles have a large depolarization in the backscattering direction but a small depolarization in the forward scattering direction. While the spherical particles do not have obvious depolarization. Therefore, depolarization in the direction of backscattering is usually regarded as an indicator for detection. If the depolarization value of the detected particle is close to 0 , it indicates that the particle tends to be spherical. In fact, particle morphologies in atmosphere are variable, so the depolarization data can be used as a powerful indicator for distinguishing the phase state of the particle. Based on phase function and depolarization of particles varying with scattering angle, the average depolarization ratio in scattering solid angle can be obtained by Eq. (9).
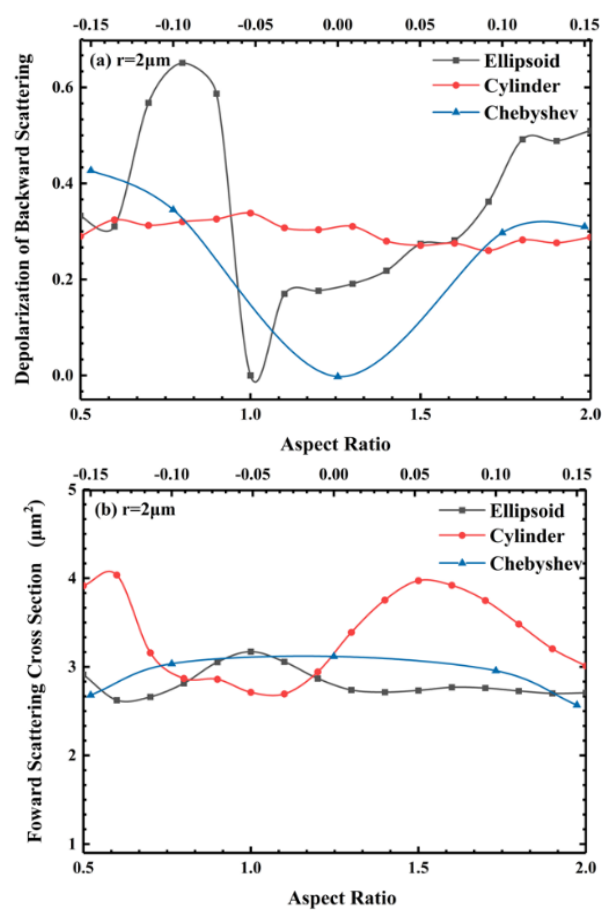

Figure 5. The relation between mean deviation of particle depolarization ratio and particle aspect ratio in receiving angle whose equivalent radius is $2 \mu \mathrm{m}$. 
Particles of different shapes have different average depolarization at different receiving angles. Figure 5a shows the relationship between the mean of the particle depolarization ratio and the aspect ratio in the backscattering main lobe region. If the aspect ratio of ellipsoid is 1 , the particle can be regarded as spherical and the depolarization is close to 0 . When the aspect ratio ranges from 0.5 to 2.0 , the depolarization of ellipsoidal particles with the same non-sphericity is smaller than that of oblate ellipsoids. The depolarization of ellipsoidal particles varies from 0 to 0.65 with a mean value of 0.35 . While that of cylindrical particles varies from 0.27 to 0.33 , with a mean value of 0.30 . Chebyshev particles with the same nonsphericity have similar depolarization values, and the range of depolarization values is 0 to 0.42 . The depolarization range of ellipsoidal particles with different shapes is larger than that of the other two kinds of particles. That is to say, when the particle size is $2 \mu \mathrm{m}$, if the depolarization values of the particles with different shapes are within the range of $0.27 \sim 0.33$ and the fluctuation is stable, the particles can be determined to be cylindrical; if the particle size is not in the range of 0.27 0.33, further analysis is needed to determine the shape of the particles. Because the depolarization of cylindrical particles with different shapes fluctuates slightly with the change of aspect ratio, it is more difficult to distinguish the specific shape of cylindrical particles by depolarization than that of ellipsoidal particles. In addition, when the aspect ratio ranges from 1.0 to 1.5 , the depolarization of the flat cylinder is basically larger than that of the flat ellipsoid.

When the aspect ratio ranges from 1.5 to 2.0 , the depolarization of the flat cylinder is basically smaller than that of the flat ellipsoid, so the ellipsoidal and cylindrical particles with the same size can be judged by depolarization data.

Figure $5 \mathrm{~b}$ shows the relationship between forward scattering cross section and aspect ratio. In the certain range for aspect ratio, three ranges of forward scattering cross sections of ellipsoidal, cylindrical and Chebyshev particles are 2.7-3.2, 2.84.0 and 2.6-3.2, respectively. The forward scattering cross section of spherical particles is larger than those of other ellipsoidal particles. For Chebyshev particles, the forward scattering cross section is larger than those of ellipsoidal particles from a holistic point of view. When the aspect ratio ranges from 0.5 to 1.0 , the forward scattering cross section of ellipsoidal particles decreases first and then increases with increasing aspect ratio. When the aspect ratio ranges from 1.0 to 2.0 , the scattering cross section of ellipsoidal particles decreases with increasing aspect ratio. Accordingly, the ellipsoidal shape in this range can be distinguished by scattering cross section. However, the scattering cross sections of long and flat cylindrical particles are similar that is difficult to be used for distinguishing the specific shape of cylindrical particles.
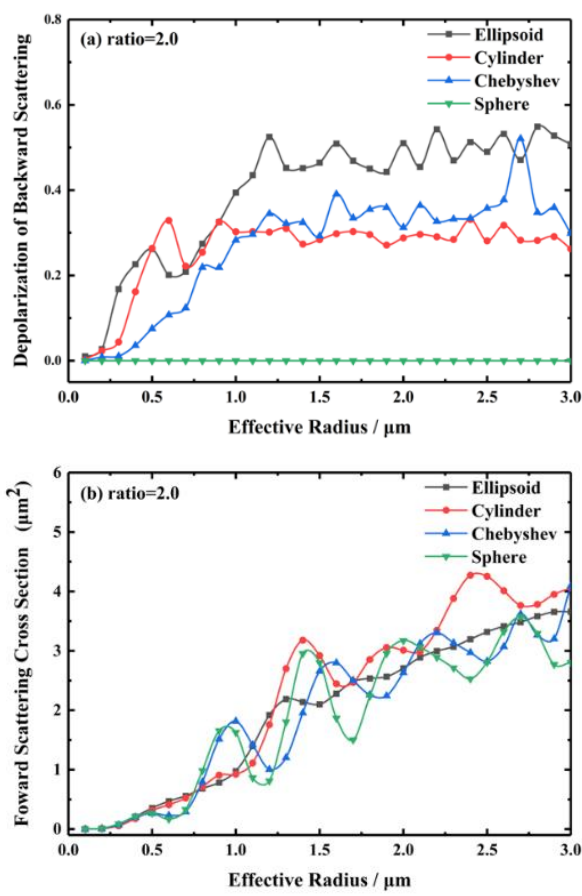

Figure 6 . The relation between mean deviation of particle depolarization ratio and particle equivalent radius in receiving angle(ratio=2).

Actually, the researches on scattering of particles are almost based on Mie scattering theory. The equivalent radius of particles is calculated according to the measured scattering cross section. Since Mie scattering theory is only applicable to spherical particles, the distribution of backscattering depolarization or forward scattering cross section of ellipsoidal, and cylindrical particles with regard to equivalent radius is calculated when the aspect ratio is 2 . The results are shown in Figure 6. Unlike the results shown in Figure 5a, depolarization fluctuates slightly with the radius of particles, showing a monotonic trend. When the equivalent radius is less than $1.0 \mu \mathrm{m}$, the difference of depolarizations is not significant; when the equivalent radius is greater than $1.0 \mu \mathrm{m}$, the depolarization ratio is greater than 0.2 , the non-spherical particles can be determined. Within a certain range of equivalent radius, the depolarization of ellipsoidal particles ranges from 0 to 0.52 , while that of cylindrical particles ranges from 0 to 0.30 , and that of ellipsoidal particles is higher than that of cylindrical particles. Therefore, the shape and size of particles can be roughly distinguished by the depolarization value when the aspect ratio is the same. In order to accurately determine the size of particles, the scattering cross section should be also considered. Figure $6 \mathrm{~b}$ reflects the relationship between the forward average scattering cross section and the equivalent radius of particles with the same shape. When the equivalent radius ranges from 0 to $3.0 \mu \mathrm{m}$, the average scattering cross section for both ellipsoidal and cylindrical particles is positively correlated with the equivalent radius, while the change of ellipsoidal particles is more stable than that of cylindrical particles. The forward scattering cross section fluctuation of spherical particles increases with the increasing particle equivalent radius. Accordingly, the method of judging particle size by forward scattering data is feasible, and the result can be more accurate if the depolarization data are considered. 


\section{CONCLUSIONS}

The scattering characteristics and aspect ratio of sulfate particles in haze are closely related to the equivalent radius of particle. The size and shape of sulfate particle can be judged by forward scattering and backward polarization. The larger the particle size is, the stronger the forward scattering is. Within a certain range of aspect ratio, the forward scattering phase function for cylindrical particle with the same particle size is the largest, followed by Chebyshev particles and ellipsoidal particles. The depolarization of spherical particles is much smaller than that of non-spherical particles. For a certain range of aspect ratio, it is more difficult to distinguish the specific shape of cylindrical particles by depolarization than that of ellipsoid particles. By comparison, however, oblate ellipsoid and oblate cylindrical particles with the same size can be distinguished obviously. The scattering cross section of spherical particles is larger than that of other ellipsoidal particles. The scattering cross section of Chebyshev particles is higher than that of ellipsoidal particles from a holistic point of view. When the equivalent radius is greater than $1.0 \mu \mathrm{m}$ and the depolarization ratio is greater than 0.2 , the non-spherical particles can be determined within a certain range of aspect ratio. The shape and size of particles can be roughly distinguished by the depolarization value under the same aspect ratio, and particle size is generally positively correlated with scattering cross section.

\section{REFERENCES}

Baumgardner, D., Nsson, H., Dawson, W., et al., 2001. The cloud, aerosol and precipitation spectrometer: a new instrument for cloud investigations. Atmospheric Research, 01, 51-264.

Bohren, C. F., Huffman, D. R., 1983. Absorption and scattering of light by small particles. New York: Wiley, 1983.

Chen, L. W., Chow, J. C., Doddridge, B. G., et al., 2003. Analysis of a summertime PM2.5 and haze episode in the midAtlantic region. Air Repair, 53(8): 946-956.

Chow, J. C., Watson, J. G., Lowenthal, D. H., et al. 2002. Comparability between PM2.5 and particle light scattering measurements, Environmental Monitoring \& Assessment, 79(1), $29-45$.

He, H., Wang, X., Wang, Y., et al., 2013. Causes and control of atmospheric haze. Bulletin of Chinese Academy of Sciences, 3(3), 344-352.

Massic, S. T., Herving, M., 2013. HITRAN 2012 refractive indices. Journal of Quantitatives Spectroscopy \& Radiative Transfer, 373-380.

Mishchenko, M. I., Travis, L. D., 1998. Capabilities and limitations of a current fortran implementation of the $\mathrm{T}$ MATRIX method for randomly oriented, rotationally symmetric scatterers. J Quant Spectrosc Ra, 60(3), 309-324.

Mishchenko, M. I., Travis, L. D., Lacis, A., 2002. Scattering, Absorption, and Emission of Light by Small Particles. Cambridge University Press, 116-117.

Mishchenko, M. I., Travis, L. D., Mackowski, D. W., 1996. Tmatrix computations of light scattering by nonspherical particles: a review. J. Quant. Spectrosc. Radiat. Transfer, 55(5), 535-575.

Tian, Y., Pan, X., Nishizawa, T., et al., 2018. Variability of depolarization of aerosol particles in Beijing mega city: implication in interaction between anthropogenic pollutants and mineral dust particles. Atmospheric Chemistry \& Physics, 1-27.

Wu, D., 2012. Summary of research on haze event in china in recent ten years. Journal of Environmental Science, 32(2): 257269.

Xu, Y. L., 2003. Scattering mueller matrix of an ensemble of variously shaped small particles. J Opt Soc Am A Opt Image Sci Vis, 20(11), 2093-2105.

Yang, F., Tan, J., Zhao, Q., et al., 2011. Characteristics of PM2.5 speciation in representative megacities and across China. Atmospheric Chemistry \& Physics, 11(11), 5207-5219.

Zhang, Y., Wang, J., Bu, L., 2019. Analysis of a haze event over Nanjing, China based on multi-source data. Atmosphere 2019,10, 338 . 